\title{
Interaction of Hyperoside with Human Serum Albumin and Effect of Glucose on the Binding
}

\author{
Jie Yang, ${ }^{1}$ Lingling Qu, ${ }^{2}$ Wenyue Yang, ${ }^{2}$ Yun Huang, ${ }^{2,3}$ Ning Jiao, ${ }^{1}$ Wenhong Zhan, \\ Ding Zhao, ${ }^{2}$ and Lijian $\mathrm{Cui}^{4}$ \\ ${ }^{1}$ The Third Hospital, Hebei Medical University, Shijiazhuang 050051, China \\ ${ }^{2}$ Pharmaceutical College, Hebei Medical University, Shijiazhuang 050017, China \\ ${ }^{3}$ Institute of Chinese Integrative Medicine, Hebei Medical University, Shijiazhuang 050017, China \\ ${ }^{4}$ Pharmaceutical College, Hebei University of Chinese Medicine, Shijiazhuang 050091, China
}

Correspondence should be addressed to Yun Huang; hy9317536@126.com and Lijian Cui; cuilijianzy@126.com

Received 21 March 2014; Revised 19 May 2014; Accepted 17 June 2014; Published 23 July 2014

Academic Editor: Austin Nevin

Copyright (C) 2014 Jie Yang et al. This is an open access article distributed under the Creative Commons Attribution License, which permits unrestricted use, distribution, and reproduction in any medium, provided the original work is properly cited.

\begin{abstract}
The interaction of hyperoside (Hyp) with human serum albumin (HSA) and effect of glucose on the binding were studied in simulating physiological condition ( $\mathrm{pH}$ 7.40). The results suggested that Hyp quenched the endogenous fluorescence of HSA via a static quenching process with the distance of $1.95 \mathrm{~nm}$ between Hyp and HSA. Hydrophobic forces played a major role in stabilizing the Hyp-HSA complex. Through synchronous fluorescence monitoring of conformation of HSA, we found that the binding to Hyp can change the microenvironment around tryptophan (Trp) residues. Increasing in glucose concentration over a range from 0 to $9 \mathrm{mM}$ decreased the binding ability of HSA to Hyp, implying that increasing in glucose concentration would increase the concentration of free Hyp.
\end{abstract}

\section{Introduction}

Hyperoside (Hyp) (Figure 1), named 2-(3,4-dihydroxyphenyl)-3-[(3R,4S,5R,6R)-3,4,5-trihydroxy-6-(hydroxymethyl)oxan-2-yl]oxy-4H-chromene-4,5,7-triol, is a fla-vonol compound isolated from the plants of Hypericaceae and Rosaceae. Hyp has a variety of physiological activities, including antioxidant, anti-inflammatory, and antiviral and so on [1-4]. In addition, Hyp plays a protective role against myocardial ischemia and cerebral ischemia injury through fighting free radicals, reducing cell apoptosis, the release of lactate dehydrogenase, and calcium inflow [5-7]. In recent years, a significant discovery has been made through the studies on the inhibitory action of Hyp on eye aldose reductase (AR) which in mammals catalyzes the conversion of glucose to sorbitol that is one of the leading causes of diabetes complications such as cataract and neurological diseases. AR inhibitory activities of $2 \mu \mathrm{M}$ Hyp were from $57.81 \%$ to $54.41 \%$ with the $50 \%$ inhibition concentration
$\left(\mathrm{IC}_{50}\right) 1.85 \pm 0.06 \mu \mathrm{M}$, which may be good for the prevention of diabetes complication [8].

Little attention has been paid on Hyp binding with human serum albumin (HSA) and the effect of glucose on HypHSA binding even though the interaction of Hyp with bovine serum albumin was reported [9]. HSA is the most abundant plasma protein in humans and acts as a transport protein and plasma carrier by nonspecific binding. As one of the most extensively studied model protein, HSA has high affinity to many endogenous and exogenous compounds, serving in transporting drugs or other organic molecules to their targets $[10,11]$.

The concentration of blood glucose is the amount of glucose present in the blood of a human or animal. Normally in human, the body maintains the blood glucose level at 3.9-6.1 mM (fasting plasma glucose) and 3.9-7.8 mM (postprandial blood glucose), above or below which may be an indicator of a medical condition [12]. Our previous research showed that blood glucose affected the binding between some 
<smiles></smiles>

Hyperoside

Figure 1: The structure of hyperoside (Hyp).

flavonol compounds and BSA [13]. It is very informative to investigate on the binding of Hyp to HSA in the presence and absence of glucose.

In order to further explore whether the interactions between Hyp and HSA occurred and the influence of glucose on them, we primarily focus on investigating the effect of Hyp on the fluorescence spectrum of HSA, the fluorescence quenching mechanism, the binding constant, the number of binding sites, the binding mode, and the effect of different glucose concentrations including $0,3,6$, or $9 \mathrm{mM}$ on the binding. These parameters are expected to provide more information about the interaction between Hyp and HSA. The studies on intermolecular interaction of Hyp with HSA and the influence of glucose on the binding will contribute to the understanding of the functions and the metabolic processes of Hyp. Finally, the results may provide an important theoretical support for the use of Hyp in the treatment of diabetic cataract.

\section{Materials and Methods}

2.1. Materials and Apparatus. HSA purchased from Sigma (New York, USA) (lyophilized powder) was used without further purification and the powder (a molecular mass $66000 \mathrm{Da}$ ) was $98-99 \%$ pure (agarose gel electrophoresis), which was prepared to the experimental concentration according to weight and the molecular mass. Tris (no less than $99.5 \%$ pure) was also from Sigma. Hyp (no less than $98 \%$ pure) was bought from national institute for the control of pharmaceutical and biological products (Beijing, China). All other chemicals were of analytical reagent grade.

Fluorescence measurements were performed with a Perkin-Elmer spectrofluorometer Model LS-55 equipped with a $150 \mathrm{~W}$ Xenon lamp and a thermostat bath. The widths of both the excitation slit and emission slit were set at $5.0 \mathrm{~nm}$ with a nominal resolution of $0.5 \mathrm{~nm}$. The absorption spectra were measured on a Shimadzu UV-2450 UV-vis spectrophotometer. The $\mathrm{pH}$ measurements were carried out on a PHS-3C digital $\mathrm{pH}$ meter. Titrations were done manually by using trace syringes. A quartz cell of $1.00 \mathrm{~cm}$ path length was used for the measurements.
2.2. Procedures. $1 \times 10^{-5} \mathrm{M}$ and $3 \times 10^{-3} \mathrm{M}$ Hyp stock solutions were prepared in methanol and $1 \times 10^{-5} \mathrm{M}$ HSA stock solution in Tris- $\mathrm{HCl}$ buffer. The stock solution was diluted with $5 \times 10^{-2} \mathrm{M}$ Tris-HCl buffer solution ( $\mathrm{pH}$ 7.4) to some lower concentration containing $5 \times 10^{-2} \mathrm{M} \mathrm{NaCl}$ for actual use.

Three mL HSA $\left(1 \times 10^{-6} \mathrm{M}\right)$ was titrated with $0,1,2$, $3,4,5,6$, or $7 \mu \mathrm{L}$ Hyp $\left(3 \times 10^{-3} \mathrm{M}\right)$, respectively, and a certain amount of Tris- $\mathrm{HCl}$ buffer solution to give the final volume of $3.01 \mathrm{~mL}$. Another solution containing HSA in the absence and presence of glucose $(3,6$, or $9 \mathrm{mM})$ was prepared similarly. The final concentration of Hyp varied from 0 to $7 \times$ $10^{-6} \mathrm{M}$ at an increment of $1 \times 10^{-6} \mathrm{M}$. The resultant mixture was subsequently ultrasonicated for $1 \mathrm{~min}$ and incubated for $5 \mathrm{~min}$ at 298,304 , or $310 \mathrm{~K}$.

Fluorescence spectra were recorded at 298 and $310 \mathrm{~K}$ in the range from 300 to $420 \mathrm{~nm}$ at excitation wavelength $280 \mathrm{~nm}$. The synchronous fluorescence spectra were recorded when $\Delta \lambda=60 \mathrm{~nm}$. The three-dimensional fluorescence spectra were performed under the following conditions: emission wavelengths over a range of $280-420 \mathrm{~nm}$, excitation at $260 \mathrm{~nm}$, scanning number 13 , and increment $5 \mathrm{~nm}$. The UV-vis absorption spectrum of Hyp (HSA or Hyp-HSA) was recorded in the range from 300 to $420 \mathrm{~nm}$ using UV-2450.

2.3. Correction of Fluorescence Intensity. Stern-Volmer equation $[14,15]$ was used to deduce HSA quenching mechanism caused by Hyp; a correction for the inner filter effect $[16,17]$ was made using (1). Consider

$$
F \mathcal{C}=F a \times e^{(A 1+A 2) / 2},
$$

where $F c$ and $F a$ are the fluorescence intensity corrected and observed, respectively. $A 1$ and $A 2$ are the absorption of Hyp solution at excitation and emission wavelength, respectively. All intensities of fluorescence used were corrected in this paper.

2.4. Determination of Free HSA Concentration. Binding ratio of HSA ( $\mathrm{HSA}_{\mathrm{br}}$ ) was calculated by (2).

The intrinsic fluorescence is mainly manifested by emission of Trp residues in molecule. When $\Delta \lambda=60 \mathrm{~nm}$, synchronous fluorescence intensities $\left(F^{\prime}\right)$ of HSA in the range from 0 to $1 \times 10^{-6} \mathrm{M}$ were recorded, which just show the fluorescence of Trp residues. The calibration curve was constructed, and a linear regression analysis between the relationship of $[\mathrm{HSA}]_{\text {free }} \times 10^{7}$ (HSA without Hyp) $(x)$ and $F^{\prime}(y)$ is plotted. The $[\mathrm{HSA}]_{\text {free }}$ were obtained through the plot. Consider

$$
\begin{gathered}
\mathrm{HSA}_{\mathrm{br}}=\frac{[\mathrm{HSA}]_{\text {bound }}}{[\mathrm{HSA}]_{\text {total }}}, \\
{[\mathrm{HSA}]_{\text {bound }}=[\mathrm{HSA}]_{\text {total }}-[\mathrm{HSA}]_{\text {free }} .}
\end{gathered}
$$

Then synchronous fluorescence intensities after Hyp being added were brought into the calibration curve to get the $[\mathrm{HSA}]_{\text {free }}$. Thus, $\mathrm{HSA}_{\mathrm{br}}$ can be evaluated from (2). 
2.5. Calculation of $K_{A}$ and $n$. Binding constant and the number of binding sites were calculated by (3).

When Hyp binds independently to a set of equivalent sites on a HSA, binding constant $\left(K_{A}\right)$ and the number of binding sites $(n)$ are given by the double logarithm regression equation $[15,18]$ :

$$
\log \left[\frac{F_{0}-F}{F}\right]=\log K_{A}+n \log [\text { Hyp }] .
$$

2.6. Calculation of Distance between HSA and Hyp. Distance between HSA and Hyp was obtained by the following equations [13]:

$$
\begin{gathered}
E_{C}=\frac{R_{0}^{6}}{\left(R_{0}^{6}+r^{6}\right)}, \\
E_{C}=\frac{E^{\prime}}{\mathrm{HAS}_{\mathrm{br}}}, \\
E^{\prime}=1-\frac{F}{F_{0}},
\end{gathered}
$$

where $E_{C}$ is the corrected energy transfer efficiency and $E^{\prime}$ the measured energy transfer efficiency. Consider

$$
\begin{gathered}
R_{0}^{6}=8.8 \times 10^{-25} K^{2} N^{-4} \Phi J \\
J=\frac{\left(\sum F_{\lambda} \varepsilon_{\lambda} \lambda^{4} \Delta \lambda\right)}{\sum F_{\lambda} \Delta \lambda} .
\end{gathered}
$$

$F$ is the fluorescence intensity of HSA when $C_{\mathrm{HSA}}$ : $C_{\text {Hyp }}=1: 1, F_{0}$ is the fluorescence intensity without Hyp, and $r$ is the binding distance between HSA and Hyp. $R_{0}$ is the critical distance with $K^{2}$ (the spatial orientation factor of the dipole $)=2 / 3, N$ (the refractive index of the medium $)=1.34$, and $\Phi$ (the fluorescence quantum yield of HSA) $=0.12$, when the transfer efficiency is $50 \%$. $J$ is the overlap integral of the fluorescence emission spectrum of HSA and the absorption spectrum of Hyp. $F_{\lambda}$ is the fluorescence intensity of HSA at wavelength $\lambda, \varepsilon_{\lambda}$ is the molar absorptivity of Hyp at wavelength $\lambda$, and $\Delta \lambda$ is wavelength span.

2.7. Binding Forces. The interaction forces between Hyp and HSA were estimated by the relationships between the thermodynamic parameters; according to the enthalpy change $\left(\Delta H^{\circ}\right)$ and entropy change $\left(\Delta S^{\circ}\right)$ before and after reaction, the main interaction forces can be estimated. Based on the binding constants at different temperatures, the free energy change $\left(\Delta G^{\circ}\right)$ can be estimated $[14,15]$. Consider

$$
\begin{gathered}
\ln \frac{K_{2}}{K_{1}}=\left(\frac{1}{T_{1}}-\frac{1}{T_{2}}\right) \frac{\Delta H^{\circ}}{R} \\
\Delta G^{\circ}=-R T \ln K \\
\Delta G^{\circ}=\Delta H^{\circ}-T \Delta S^{\circ},
\end{gathered}
$$

where $K$ is the binding constant at the corresponding experimental temperature $T$ and $R$ is the gas constant.

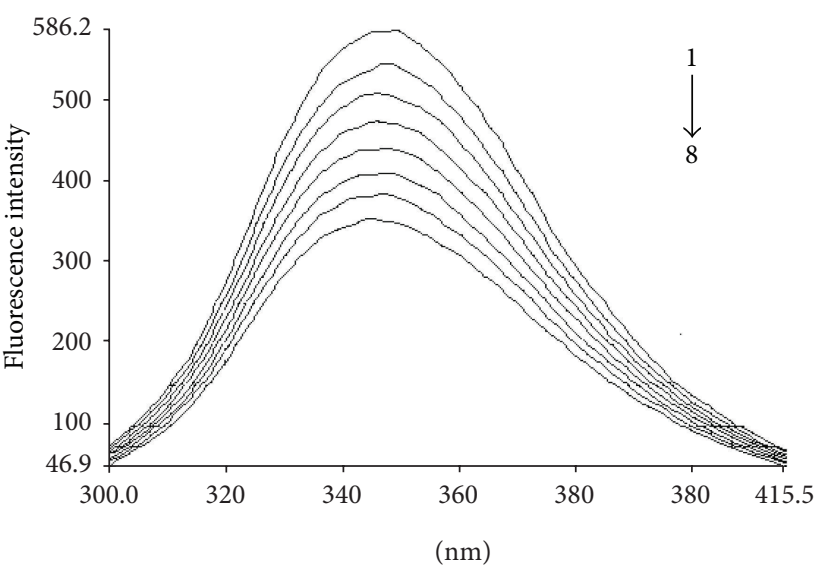

FIGURE 2: The fluorescence quenching spectra of HSA at different concentration of Hyp $\left(T=298 \mathrm{~K}, \mathrm{pH}=7.4\right.$ and $\left.\lambda_{\text {ex }}=280 \mathrm{~nm}\right)$; from curve 1 to $8, C_{\mathrm{HSA}}=1.0 \times 10^{-6} \mathrm{M}, C_{\mathrm{Hyp}}=0,1,2,3,4,5,6$, and $7 \times$ $10^{-6} \mathrm{M}$, respectively.

\section{Results and Discussion}

3.1. Effect of Hyp on Fluorescence Spectrum of HSA. The study shows that the peak shape and intensity of HSA fluorescence emission were very stable and highly repeatable in Tris$\mathrm{HCl}$ buffer solution and not affected by a small amount of methanol.

At excitation wavelength $280 \mathrm{~nm}$, scanning fluorescence spectra showed that HSA had a strong fluorescence emission peak at $349 \mathrm{~nm}$ and Hyp did not. Because Hyp has weak UV absorption at 280 and $349 \mathrm{~nm}$, (1) was used to correct for the inner filter effects caused by the absorption of Hyp.

HSA emission spectrum is the reflection of the kind, structure, and microenvironment of fluorescence chromophore, mainly including Trp and Tyr residues [19], through which drug-protein complex is usually studied. The maximum emission wavelength of HSA, used to judge whether the protein conformation is changed, is closely related to the microenvironment of aromatic residues [2022]. The fluorescence quenching spectra of HSA with different concentrations of Hyp are shown in Figure 2. The fluorescence intensities of HSA at $349 \mathrm{~nm}$ decreased regularly with the increase of Hyp concentration and the maximum hypsochromic shift was $5 \mathrm{~nm}\left(\lambda_{\max }\right.$ from 349 to $\left.344 \mathrm{~nm}\right)$ at $310 \mathrm{~K}$, suggesting that the complex of Hyp-HSA was formed, which is responsible for the quenching of HSA fluorescence.

Dynamic quenching, static quenching, and nonradiative energy transfer are three major causes of intrinsic fluorescence quenching [15]. The possible quenching mechanism can be interpreted by the fluorescence quenching spectra and the Stern-Volmer curves of Hyp with HSA. In order to confirm the view, the procedure of the fluorescence quenching was first assumed to be dynamic quenching.

The $K_{\mathrm{SV}}$ values increase with an increase in temperature for dynamic quenching, for which an increase in temperature is favorable, and the reverse effect would be observed for static quenching $[15,23]$. If the quenching type includes both static 


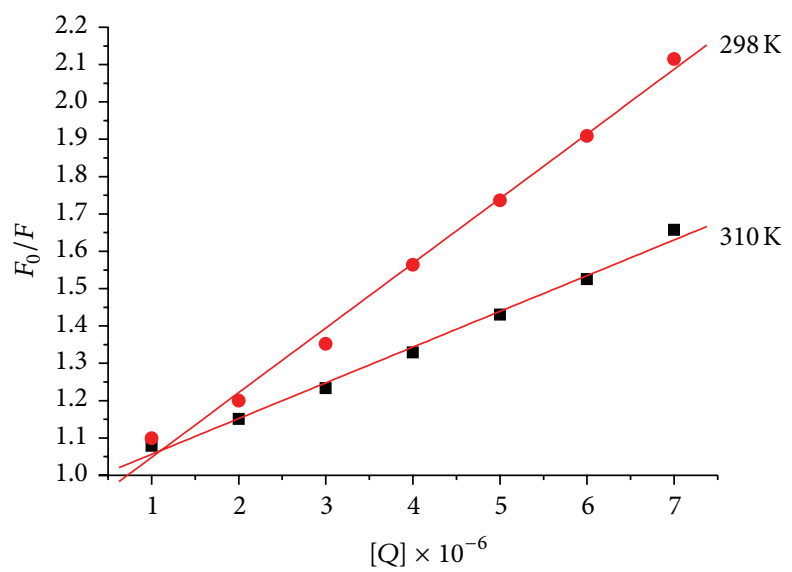

Figure 3: Stern-Volmer plots of Hyp-HSA at different temperatures, $C_{(\mathrm{Hyp})}=1,2,3,4,5,6,7 \times 10^{-6} \mathrm{M}$.

TABLE 1: Quenching rate constant of the bimolecule $\left(K_{q}\right)$, quenching constant $\left(K_{\mathrm{SV}}\right)$, binding constant $\left(K_{A}\right)$, and the number of binding sites (n) of Hyp to HAS.

\begin{tabular}{|c|c|c|c|c|c|c|c|c|c|}
\hline \multirow{2}{*}{$T(\mathrm{~K})$} & \multirow[b]{2}{*}{ Glucose concentration $\mathrm{mM}$} & \multicolumn{4}{|c|}{ Stern-Volmer equation } & \multicolumn{4}{|c|}{ Double logarithm regression equation } \\
\hline & & $\begin{array}{c}K_{q} \\
\times 10^{12} \\
\mathrm{~L} \cdot \mathrm{mol}^{-1} \mathrm{~s}^{-1}\end{array}$ & $\begin{array}{c}K_{\mathrm{SV}} \\
\times 10^{4} \\
\mathrm{~L} \cdot \mathrm{mol}^{-1}\end{array}$ & $R^{\mathrm{a}}$ & $\mathrm{SD}^{\mathrm{b}}$ & $\begin{array}{c}K_{A} \\
\times 10^{5} \\
\mathrm{~L} \cdot \mathrm{mol}^{-1}\end{array}$ & $n$ & $R^{\mathrm{a}}$ & $\mathrm{SD}^{\mathrm{b}}$ \\
\hline \multirow[t]{2}{*}{298} & 0 & 17.3 & 17.3 & 0.992 & 0.0159 & 2.47 & 1.09 & 0.996 & 0.0108 \\
\hline & 0 & 9.75 & 9.75 & 0.993 & 0.0097 & 42.7 & 1.28 & 0.995 & 0.0123 \\
\hline \multirow{3}{*}{310} & 3 & & & & & 16.2 & 1.19 & 0.997 & 0.0098 \\
\hline & 6 & & & & & 11.1 & 1.17 & 0.984 & 0.0130 \\
\hline & 9 & & & & & 3.23 & 1.10 & 0.992 & 0.0103 \\
\hline
\end{tabular}

${ }^{a}$ The correlation coefficient.

${ }^{\mathrm{b}}$ The standard deviation.

and dynamic, the Stern-Volmer plot is an upward curvature [24].

Figure 3 shows the Stern-Volmer plots of the fluorescence quenching of HSA by Hyp at different temperatures. The SV plots have good linearity with each regression coefficient above 0.99 . The results (Table 1 ) show that within the detected concentrations the $K_{\mathrm{SV}}$ is correlated with temperature and decrease from $1.73 \times 10^{5}$ to $9.75 \times 10^{4} \mathrm{~L} \cdot \mathrm{mol}^{-1}$ with the temperature increasing, which indicates that the probable quenching mechanism of Hyp-HSA is static quenching, rather than dynamic quenching [15, 25-27].

In general, for static quenching, the value of $K_{q}$ is above $2.0 \times 10^{10} \mathrm{~L} \mathrm{~mol}^{-1} \mathrm{~s}^{-1}$ [11]. $K_{q}$ initiated by Hyp $(17.3 \times$ $10^{12} \mathrm{~L} \cdot \mathrm{mol}^{-1} \mathrm{~s}^{-1}(298 \mathrm{~K})$ or $\left.9.75 \times 10^{12}(310 \mathrm{~K})\right)$ is much greater than $2.0 \times 10^{10} \mathrm{~L} \cdot \mathrm{mol}^{-1} \mathrm{~s}^{-1}$, which means that the quenching process is static.

3.2. Binding Ratio of $H S A\left(H S A_{b r}\right)$. By the linear regression analysis between $[\mathrm{HSA}]_{\text {free }} \times 10^{7}$ (without Hyp) and $F^{\prime}$ (Figure 4), (10) was obtained

$$
y=62.129 x+7.6217 \quad\left(R^{2}=0.9972\right),
$$

where $R$ is the correlation coefficient.

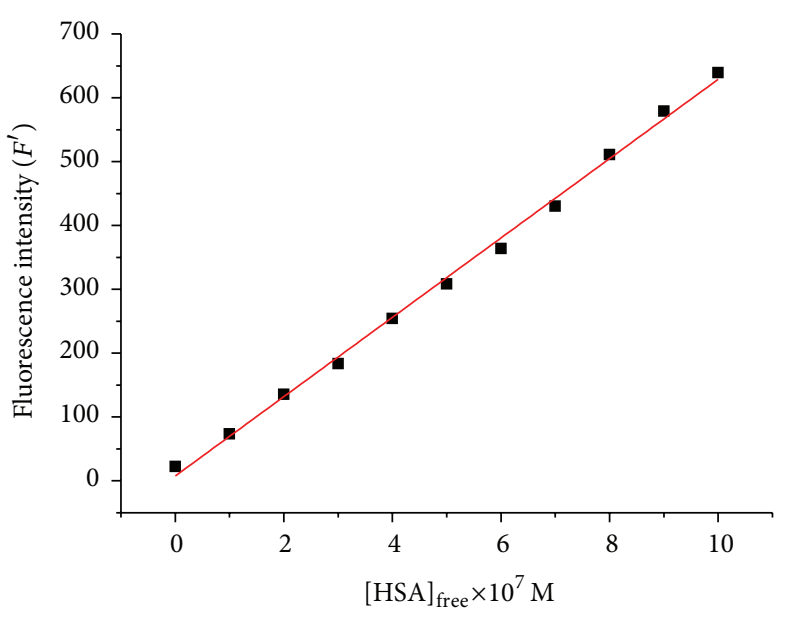

FIGURE 4: Linear regression analysis between $[\mathrm{HSA}]_{\text {free }}$ and $F^{\prime}\left(F^{\prime}\right.$ the Synchronous fluorescence intensity when $\Delta \lambda=60 \mathrm{~nm}), C_{(\mathrm{HSA})}$ $=0,1,2,3,4,5,6,7,8,9,10 \times 10^{-7} \mathrm{M}$.

When $\Delta \lambda=60 \mathrm{~nm}$, the synchronous fluorescence intensities after Hyp added were brought into (7). [HSA $]_{\text {free }}=$ 


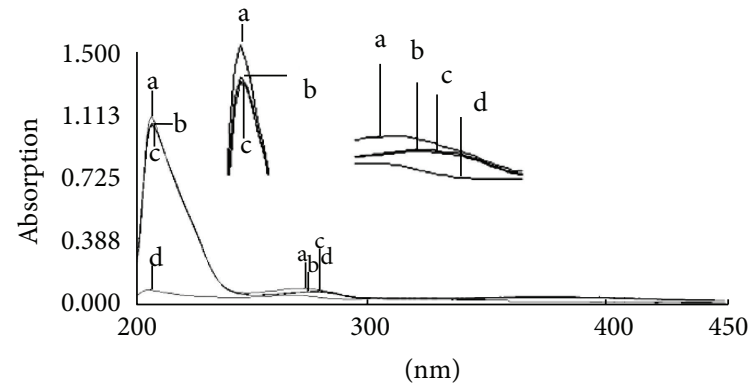

(a) $C_{(\mathrm{HSA})}=\mathrm{C}_{(\mathrm{Hyp})}=1.0 \times 10^{-6} \mathrm{M}$

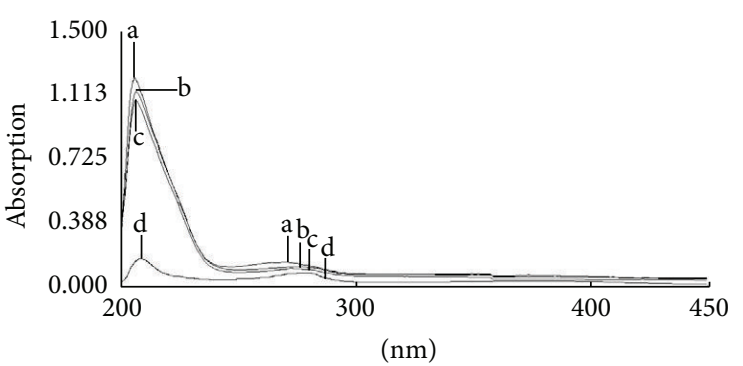

(b) $C_{(\mathrm{HSA})}=1.0 \times 10^{-6} \mathrm{M} ; \mathrm{C}_{(\mathrm{Hyp})}=2.0 \times 10^{-6} \mathrm{M}$

Figure 5: The absorption spectra of HSA, Hyp, and Hyp-HSA system. a: [Hyp-HSA], b: [HSA], and c: [Hyp-HSA]—[Hyp], d: [Hyp].

$0.96 \times 10^{-6} \mathrm{M}$ was got when $C_{\mathrm{HSA}}=C_{\mathrm{Hyp}}=1 \times 10^{-6} \mathrm{M}$; thus, $\mathrm{HSA}_{\mathrm{br}}=4 \%$ from (2).

HSA $_{\text {br }}$ was $7.9 \%$ when $C_{\mathrm{HSA}}: C_{\mathrm{Hyp}}=1: 2$.

3.3. Effect of Hyp on UV Absorption of HSA. The structural change and the complex formation between Hyp and HSA can also be explored by UV absorption measurement. For static quenching, due to forming nonfluorescent complexes with fluorophores in the ground state, the absorption spectra of HSA with quencher are different from those without quencher, while for dynamic quenching affecting the excitation state of fluorophores does not change the absorption spectra of HSA [15, 23-25, 28].

The absorption spectra of HSA in the absence and presence of Hyp were recorded by subtracting the corresponding spectrum of Hyp free form in the buffer from that of HypHSA system in Figure 5. HSA has two main peaks (210 and $278 \mathrm{~nm}$ ) in its absorption spectrum, which shows that the addition of Hyp made the absorption peak of HSA very small decrease at $210 \mathrm{~nm}$ and no significant changes at $278 \mathrm{~nm}$ when $\mathrm{HSA}_{\mathrm{br}}$ was 4\% (Figure 5(a)) and significant drop at $210 \mathrm{~nm}$ and $278 \mathrm{~nm}$ when $\mathrm{HSA}_{\text {br }}$ was $7.9 \%$ (Figure 5(b)). The absorption spectral changes of HSA in the presence of Hyp strongly suggest the complex formation between Hyp and HSA.

3.4. Binding Constant and the Number of Binding Sites. From Figure 6, the double logarithm regression curves possessed good linearity and the slope significantly increased with temperature increasing.

The values of $K_{A}$ and $n$ of Hyp binding to HSA increased with temperature increasing from $2.47 \times 10^{5}$ to $4.27 \times$ $10^{6} \mathrm{~L} \cdot \mathrm{mol}^{-1}$ and from 1.09 to 1.28 , respectively.

3.5. Distance between HSA and Hyp. Figure 7 shows the overlapping plot between absorption spectrum of Hyp and emission spectrum of HSA. The value of $J$ is evaluated from (8), which was $J=2.67 \times 10^{-14} \mathrm{~cm}^{3} \cdot \mathrm{L} \cdot \mathrm{mol}^{-1}$. Thus, we obtained that $R_{0}=3.00 \mathrm{~nm}$ from (7); $E^{\prime}=0.04$ from (6).

When $C_{\mathrm{HSA}}=C_{\mathrm{Hyp}}=1 \times 10^{-6} \mathrm{~mol} \mathrm{~L}^{-1}$ and HSA binding ratio was calculated to be $4 \%$, then, $E_{C}=0.93$ was obtained from (5).

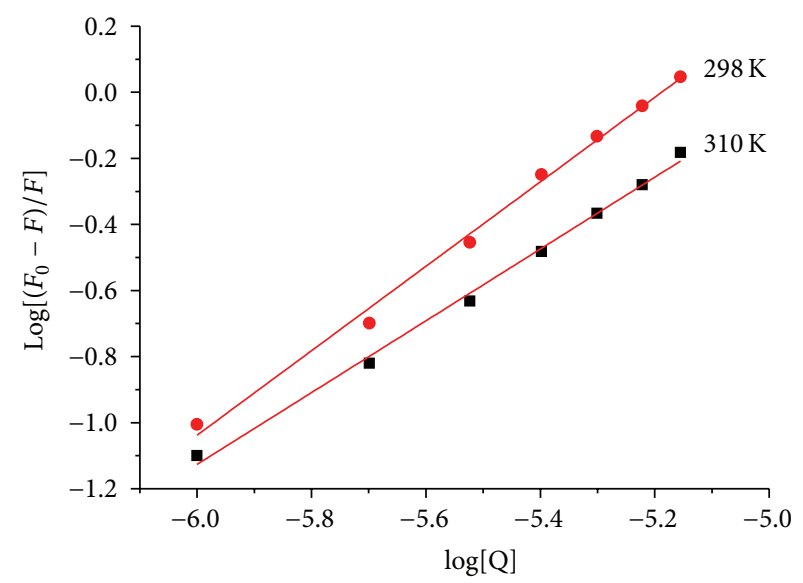

FIGURE 6: The double logarithm regression plots of Hyp-HSA at different temperatures, $C_{(\mathrm{HSA})}=1,2,3,4,5,6,7 \times 10^{-6} \mathrm{M}$.

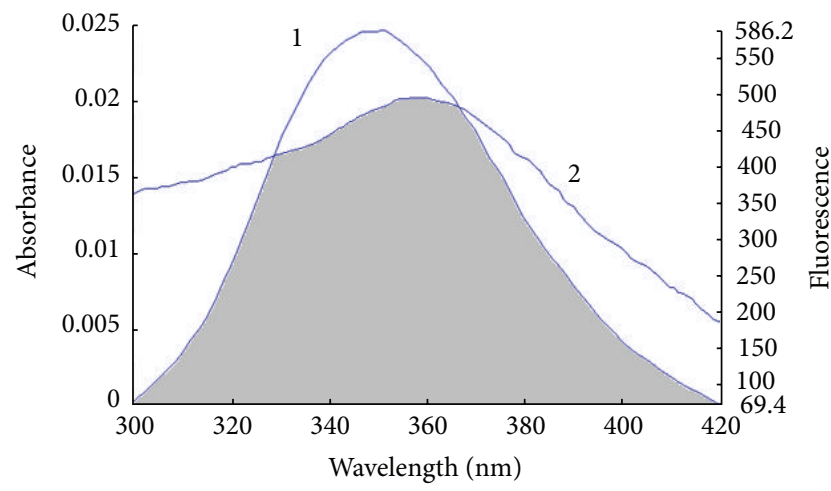

FIGURE 7: Overlap of fluorescence emission spectrum of HSA (curve 1) and UV absorption spectrum of Hyp (curve 2); $C_{(\mathrm{Hyp})}=C_{(\mathrm{HSA})}=$ $1 \times 10^{-6} \mathrm{M}$.

The distance $(r)$ was $1.95 \mathrm{~nm}$ from (4).

3.6. Binding Mode. The interaction forces between Hyp and HSA mainly involve hydrogen bonds, Van der Waals forces, electrostatic interactions and hydrophobic forces, and so forth $[14,15]$. 
TABLE 2: Thermodynamic parameters of the interaction between Hyp and HAS.

\begin{tabular}{lccc}
\hline$T / \mathrm{K}$ & $\Delta H^{\circ} /\left(\mathrm{kJ} \cdot \mathrm{mol}^{-1}\right)$ & $\Delta S^{\circ} /\left(\mathrm{J} \cdot \mathrm{mol}^{-1} \mathrm{~K}^{-1}\right)$ & $\Delta G^{\circ} /\left(\mathrm{kJ} \cdot \mathrm{mol}^{-1}\right)$ \\
\hline $298 \mathrm{~K}$ & 36.70 & 226.37 & -30.76 \\
$304 \mathrm{~K}$ & 36.70 & 227.39 & -29.75 \\
$310 \mathrm{~K}$ & 36.70 & 227.61 & -29.28 \\
\hline
\end{tabular}

From the experimental data shown in Table 2, the thermodynamic parameters of Hyp binding to HSA were $\Delta H^{\circ}>$ 0 and $\Delta S^{\circ}>0$, which indicated that the predominant intermolecular forces to stabilize the complex of Hyp-HSA were hydrophobic forces. The negative $\Delta G^{\circ}$ value was taken as evidence for the spontaneity of the binding of Hyp with HSA at standard conditions.

3.7. Synchronous Fluorescence Spectra. The intrinsic fluorescence of HSA is manifested by emission of Trp and Tyr residues in molecule [19]. When $\Delta \lambda=60 \mathrm{~nm}$, synchronous fluorescence spectra just showed the fluorescence of Trp residues, the maximum emission wavelength of which is related to its microenvironment $[14,15]$.

The synchronous fluorescence spectra of HSA (Figure 8) show that the fluorescence intensity of HSA at $285 \mathrm{~nm}$ decreased regularly and the maximum emission wavelength had weak bathochromic shift (from 285 to $287 \mathrm{~nm}$ ) with a fixed amount of HSA and the increase of Hyp concentration, which represented that the conformation of HSA was somewhat changed leading to decreased polarity around the Trp residues.

3.8. Influence of Glucose on Binding Constant, the Number of Binding Sites, and Conformation. In order to investigate the influence of glucose on the interaction of Hyp with HSA, the binding constants and the number of binding sites in presence and absence of different concentrations of glucose $(3,6$, or $9 \mathrm{mM})$ at $310 \mathrm{~K}$ were compared. Glucose decreased binding constant and the number of binding sites of Hyp with $\mathrm{HSA}$ at $310 \mathrm{~K}\left(K_{A}\right.$ from $4.27 \times 10^{6}$ to $1.62 \times 10^{6}, 1.11$ $\times 10^{6}$ and $3.23 \times 10^{5} \mathrm{~L} \cdot \mathrm{mol}^{-1}$; $n$ from 1.28 to $1.19,1.17$, and 1.10, resp.) (Table 1). The double logarithm curves possessed good linearity and the slope significantly decreased with the increase of sugar level (Figure 9).

The three-dimensional fluorescence spectra of Hyp-HSA in the absence and presence of glucose were shown in Figure 10, respectively.

The excitation wavelength, emission wavelength and fluorescence intensity as axes in the fluorescence emission spectra, three-dimensional fluorescence technique is commonly used for the investigation of conformational changes of proteins interacting with drugs $[29,30]$. It was reported that the three-dimensional fluorescence spectrum is an effective means to investigate the characteristic conformational change of protein [31-33].

Two fluorescence peaks 1 and 2 were easily observed in three-dimensional fluorescence spectra. Peak 1 is the Rayleigh scattering peak $\left(\lambda_{\mathrm{ex}}=\lambda_{\mathrm{em}}\right)$. Peak $2\left(\lambda_{\mathrm{ex}}<\lambda_{\mathrm{em}}, \Delta \lambda>0\right)$

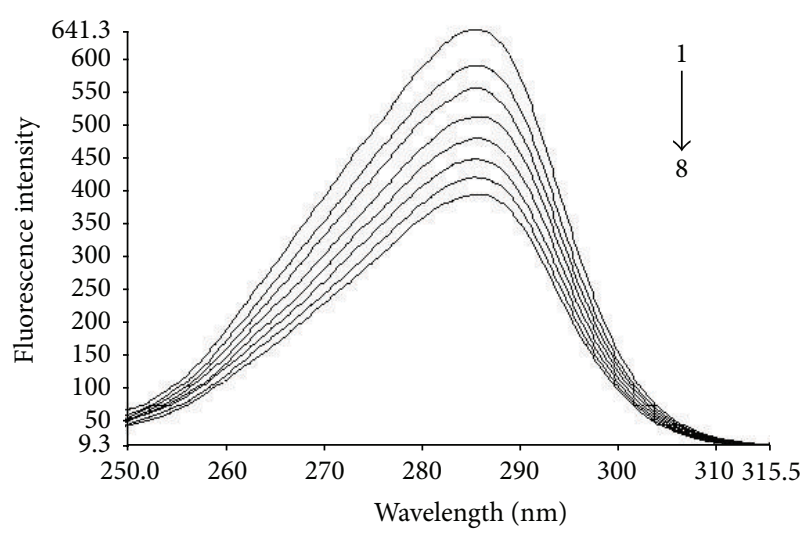

FIGURE 8: Synchronous fluorescence spectra of interaction between HSA and Hyp; from curve 1 to $8, C_{(\mathrm{HSA})}=1 \times 10^{-6} \mathrm{M}, C_{(\mathrm{Hyp})}=0,1$, $2,3,4,5,6$, and $7 \times 10^{-6} \mathrm{M}$, respectively.

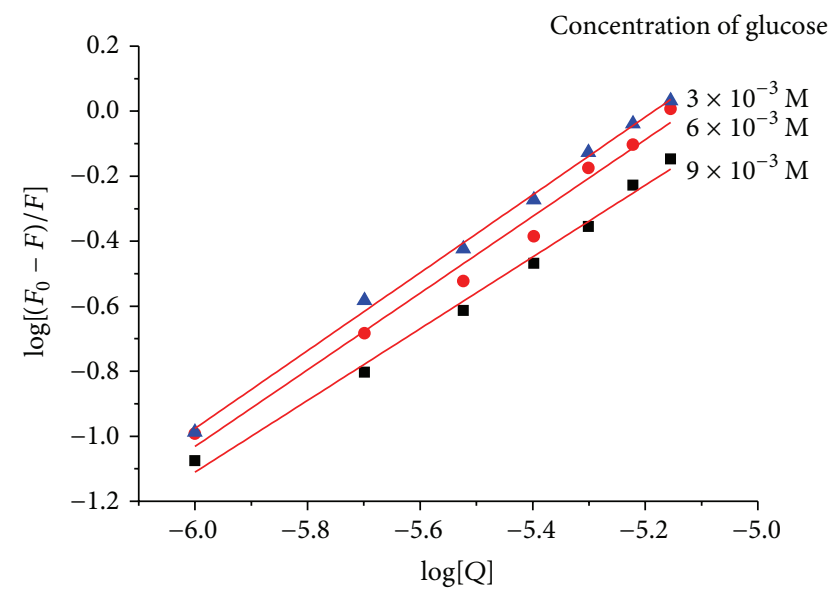

FIGURE 9: The double logarithm regression plots of Hyp-HSA at different concentrations of glucose.

which reveals the spectral behavior of Trp and Tyr residues, of which the maximum emission wavelength and fluorescence intensity show a close relation to the polarity of their microenvironment, was the primary fluorescence peak we studied $[34,35]$.

The fluorescence intensity of Hyp-HSA in the presence of glucose (at 433,426 , and 415 , resp.) was weaker than that $(446 \mathrm{~nm})$ without glucose.

By comparison, it could be seen that all the maximum excitation wavelengths were at $280 \mathrm{~nm}$ and the maximum excitation wavelengths were from 347 to $342 \mathrm{~nm}$ in the threedimensional fluorescence spectra (Figures 10(a), 10(b), 10(c), $10(\mathrm{~d})$, and $10(\mathrm{e}))$ and the Stokes shift from 67 to $62 \mathrm{~nm}(\Delta \lambda$ between excitation wavelength and emission wavelength) changed which demonstrated that glucose had an effect on the polarity and hydrophilicity around Trp microenvironment [33].

By decreasing the binding constant and the number of binding sites of Hyp with HSA, glucose could improve the plasma concentration of free Hyp, which may be help for 


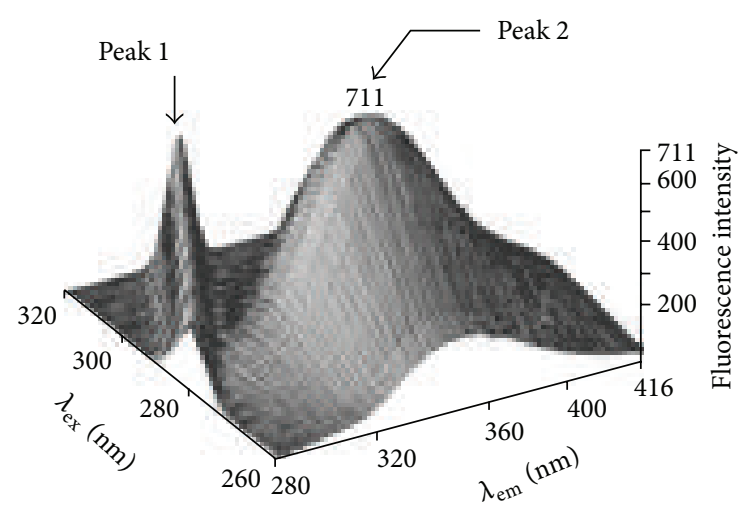

(a)

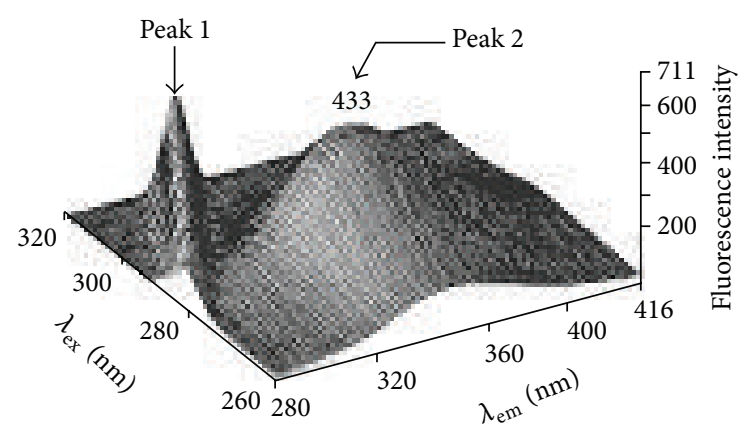

(c)

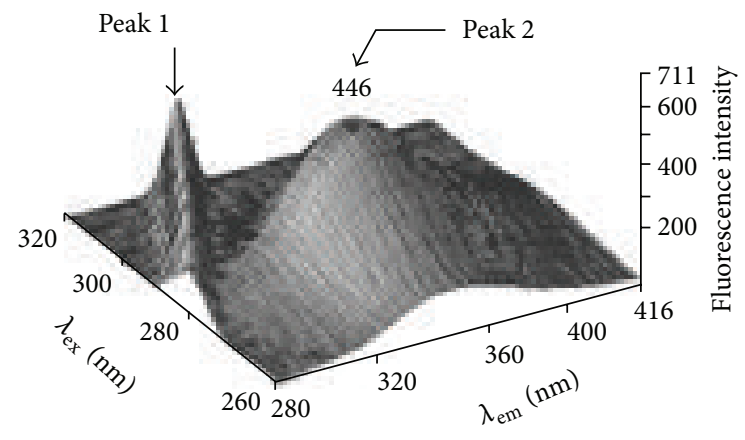

(b)

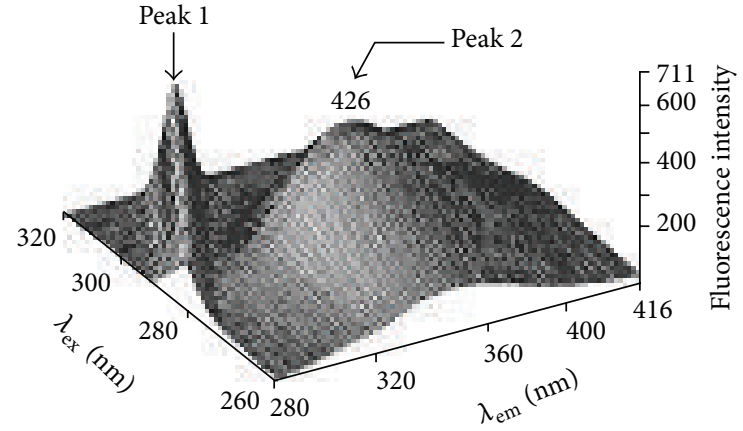

(d)

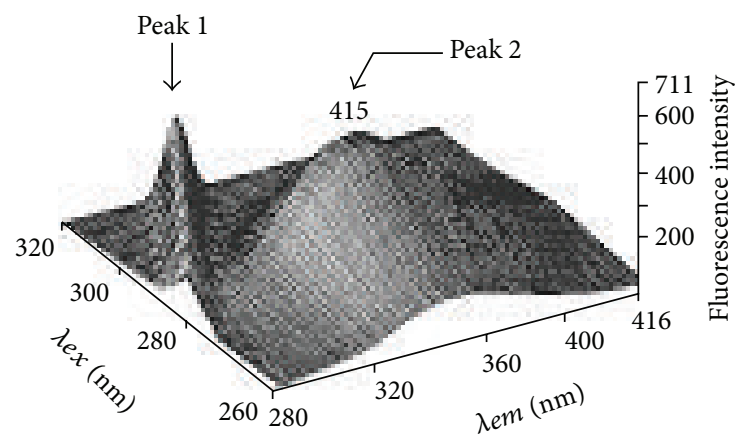

(e)

Figure 10: Three-dimensional fluorescence spectra of HSA (a), Hyp-HSA without glucose (b), Hyp-HSA with 3 mM glucose (c), Hyp-HSA with $6 \mathrm{mM}$ glucose (d), and Hyp-HSA with $9 \mathrm{mM}$ glucose (e) at $310 \mathrm{~K} ; C_{(\mathrm{HSA})}=1 \times 10^{-6} \mathrm{M}, C_{(\mathrm{Hyp})}=7 \times 10^{-6} \mathrm{M}$.

the inhibitory action on eye aldose reductase and diabetic cataract.

\section{Conclusions}

This paper presents the interaction of Hyp with HSA and the effect of glucose on the binding by equilibrium UVvis absorption, fluorescence emission, synchronous fluorescence, and three-dimensional fluorescence spectra.

It was shown that Hyp quenched the endogenous fluorescence of HSA via a static quenching procedure. The distance between Hyp and HAS was $1.95 \mathrm{~nm}$. Hydrophobic forces played a major role in stabilizing the complex of Hyp-HSA. The conformation and microenvironment of HSA were changed after Hyp binding to HSA. By decreasing $K_{A}$ and $n$ of Hyp to HSA, glucose can elevate blood drug level of Hyp, from which we can deduce that Hyp may have stronger biological activity in diabetics than that in normal people. The binding constants decreased with sugar concentration increasing, which indicate concentration of free Hyp rising that is advantageous to prevent of diabetic cataract.

\section{Conflict of Interests}

The authors declare that there is no conflict of interests regarding the publication of this paper. 


\section{Acknowledgment}

The authors gratefully acknowledge financial support from the HEBEI Province Science and Technology Support Program under Grant no. 142777114D (Insulin and traditional Chinese medicine injection in treating diabetes and its complications-clinical observation on curative effect and mechanism analysis).

\section{References}

[1] L. Luo, Q. Sun, Y. Y. Mao, Y. H. Lu, and R. X. Tan, "Inhibitory effects of flavonoids from Hypericum perforatum on nitric oxide synthase," Journal of Ethnopharmacology, vol. 93, no. 2-3, pp. 221-225, 2004.

[2] M. J. Piao, K. A. Kang, R. Zhang et al., "Hyperoside prevents oxidative damage induced by hydrogen peroxide in lung fibroblast cells via an antioxidant effect," Biochimica et Biophysica Acta: General Subjects, vol. 1780, no. 12, pp. 1448-1457, 2008.

[3] Z. Liu, X. Tao, C. Zhang, Y. Lu, and D. Wei, "Protective effects of hyperoside (quercetin-3-o-galactoside) to PC12 cells against cytotoxicity induced by hydrogen peroxide and tertbutyl hydroperoxide," Biomedicine and Pharmacotherapy, vol. 59, no. 9, pp. 481-490, 2005.

[4] L. Wu, X. Yang, Z. Huang, H. Liu, and G. Wu, "In vivo and in vitro antiviral activity of hyperoside extracted from Abelmoschus manihot (L) medik," Acta Pharmacologica Sinica, vol. 28, no. 3, pp. 404-409, 2007.

[5] Z. Chen, J. Zhang, and C. Ma, "Protective effect of hyperin on cerebral infarction in rats," China Journal of Chinese Materia Medica, vol. 23, no. 10, pp. 626-628, 1998.

[6] Z. W. Chen, C. G. Ma, and W. Z. Zhao, "Protective effect of hyperin against cerebral ischemia-reperfusion injury," Acta Pharmaceutica Sinica, vol. 33, no. 1, pp. 14-17, 1998.

[7] Q.-L. Li, G.-X. Chou, Z.-W. Chen, and C.-G. Ma, "Inhibitory mechanism of hyperin on the apoptosis in myocardial ischemia/reperfusion in rats ," Acta Pharmaceutica Sinica, vol. 37, no. 11, pp. 849-852, 2002.

[8] H. A. Jung, M. D. N. Islam, Y. S. Kwon et al., "Extraction and identification of three major aldose reductase inhibitors from Artemisia montana," Food and Chemical Toxicology, vol. 49, no. 2, pp. 376-384, 2011.

[9] L. Man-Xiu and D. Yan-Zhi, "Investigation on the interaction of hyperoside and bovine serum albumin," Chinese Journal of Analysis Laboratory, vol. 30, no. 5, pp. 46-49, 2011.

[10] Y. Huang, L. J. Cui, Y. H. Dou, and Y. L. Wang, "Research on the interaction of mechanism between aspirin and human serum albumin," Chinese Pharmacological Bulletin, vol. 24, no. 9, pp. 1192-1195, 2008.

[11] É. A. Enyedy, E. Farkas, O. Dömötör, and M. A. Santos, "Interaction of folic acid and some matrix metalloproteinase (MMP) inhibitor folate- $\gamma$-hydroxamate derivatives with $\mathrm{Zn}$ (II) and human serum albumin," Journal of Inorganic Biochemistry, vol. 105, no. 3, pp. 444-453, 2011.

[12] M. G. Valdovinos and D. Weyand, "Blood glucose levels and problem behavior," Research in Developmental Disabilities, vol. 27, no. 2, pp. 227-231, 2006.

[13] Y. Huang, L. J. Cui, C. Chen, Y. H. Dou, W. H. Zhan, and Y. L. Wang, "The comparative studies on the interaction of baicalein and baicalin with bovine serum albumin and the influence of glucose," Chinese Pharmacological Bulletin, vol. 26, no. 6, pp. 754-758, 2010.
[14] J. G. Xu and Z. B. Wang, Methods of Fluorescence Analysis, Science Press, Beijing, China, 3rd edition, 2006.

[15] J. R. Lakowicz, Principles of Fluorescence Spectroscopy, Plenum Press, New York, NY, USA, 3rd edition, 2006.

[16] J. R. Lakowicz and G. Weber, "Quenching of protein fluorescence by oxygen. Detection of structural fluctuations in proteins on the nanosecond time scale," Biochemistry, vol. 12, no. 21, pp. 4161-4170, 1973.

[17] B. Birdsall, R. W. King, and M. R. Wheeler, "Correction for light absorption in fluorescence studies of protein-ligand interactions," Analytical Biochemistry, vol. 132, no. 2, pp. 353361, 1983.

[18] M. Van De Weert and L. Stella, "Fluorescence quenching and ligand binding: a critical discussion of a popular methodology," Journal of Molecular Structure, vol. 998, no. 1-3, pp. 145-150, 2011.

[19] B. Tang, M. Du, Z. Z. Chen, H. Zhang, and H. X. Shen, "Studies on luminescence of Trp and Tyr residues in protein denaturation by three-dimensional-synchronous-polarized spectrofluorimetry," Acta Chimica Sinica, vol. 62, no. 12, pp. 1153-1157, 2004.

[20] P. Qu, H. Lu, X. Y. Ding, Y. Tao, and Z. H. Lu, "Study on the interaction of 6-thioguanine with bovine serum albumin by spectroscopic techniques," Journal of Molecular Structure, vol. 920, no. 1-3, pp. 172-177, 2009.

[21] G. W. Zhang, X. X. Chen, J. B. Guo, and J. J. Wang, "Study on the interaction of hesperidin or icariin with lysozyme by fluorescence spectroscopy," Spectroscopy and Spectral Analysis, vol. 29, no. 1, pp. 184-187, 2009.

[22] D. Li, J. Zhu, and J. Jin, "Spectrophotometric studies on the interaction between nevadensin and lysozyme," Journal of Photochemistry and Photobiology A: Chemistry, vol. 189, no. 1, pp. 114-120, 2007.

[23] J. Chen, X. Y. Jiang, X. Q. Chen, and Y. Chen, "Effect of temperature on the metronidazole-BSA interaction: multispectroscopic method," Journal of Molecular Structure, vol. 876, no. 1-3, pp. 121-126, 2008.

[24] Y.-Q. Wang, H.-M. Zhang, and Q.-H. Zhou, "Studies on the interaction of caffeine with bovine hemoglobin," European Journal of Medicinal Chemistry, vol. 44, no. 5, pp. 2100-2105, 2009.

[25] E. H. Liu, L. W. Qi, and P. Li, "Structural relationship and binding mechanisms of five flavonoids with bovine serum albumin," Molecules, vol. 15, no. 12, pp. 9092-9103, 2010.

[26] M. Liang, R. Liu, W. Qi et al., "Interaction between lysozyme and procyanidin: multilevel structural nature and effect of carbohydrates," Food Chemistry, vol. 138, no. 2-3, pp. 1596-1603, 2013.

[27] S. Bi, P. Bo, T. Wang, T. Zhao, and W. Yu, "Investigation on the interactions of clenbuterol to bovine serum albumin and lysozyme by molecular fluorescence technique," Spectrochimica Acta Part A: Molecular and Biomolecular Spectroscopy, vol. 120, no. 24, pp. 456-461, 2014.

[28] H. Zhang, Y. Xu, Q. Zhou, and Y. Wang, "Investigation of the interaction between chlorophenols and lysozyme in solution," Journal of Photochemistry and Photobiology B: Biology, vol. 104, no. 3, pp. 405-413, 2011.

[29] Y. Huang, L. Cui, J. Wang et al., "Interaction of aconitine with bovine serum albumin and effect of atropine sulphate and glycyrrhizic acid on the binding," Journal of Luminescence, vol. 132, no. 2, pp. 357-361, 2012. 
[30] T. Wang, Z. Zhao, B. Wei, L. Zhang, and L. Ji, "Spectroscopic investigations on the binding of dibazol to bovine serum albumin," Journal of Molecular Structure, vol. 970, no. 1-3, pp. 128-133, 2010.

[31] G. Zhang, Q. Que, J. Pan, and J. Guo, "Study of the interaction between icariin and human serum albumin by fluorescence spectroscopy," Journal of Molecular Structure, vol. 881, no. 1-3, pp. 132-138, 2008.

[32] H. von der Dick and W. Kalkreuth, "Synchronous excitation and three-dimensional fluorescence spectroscopy applied to organic geochemistry," Organic Geochemistry, vol. 10, no. 1-3, pp. 633639,1986

[33] F. Ding, G. Zhao, J. Huang, Y. Sun, and L. Zhang, "Fluorescence spectroscopic investigation of the interaction between chloramphenicol and lysozyme," European Journal of Medicinal Chemistry, vol. 44, no. 10, pp. 4083-4089, 2009.

[34] Y. Huang, L. Cui, J. Wang et al., "Comparative studies on interactions of baicalein, baicalin and scutellarin with lysozyme," European Journal of Medicinal Chemistry, vol. 46, no. 12, pp. 6039-6045, 2011.

[35] X. Hu, S. Cui, and J. Q. Liu, "Fluorescence studies of interaction between flavonol p-coumaroylglucoside tiliroside and bovine serum albumin," Spectrochimica Acta-Part A: Molecular and Biomolecular Spectroscopy, vol. 77, no. 2, pp. 548-553, 2010. 

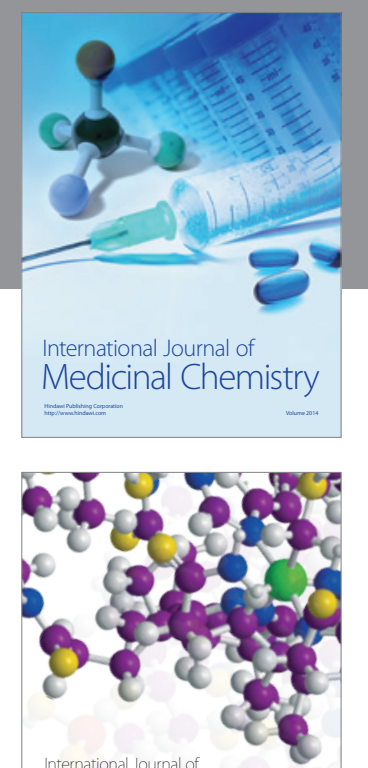

\section{Carbohydrate} Chemistry

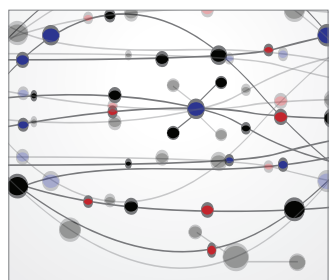

The Scientific World Journal
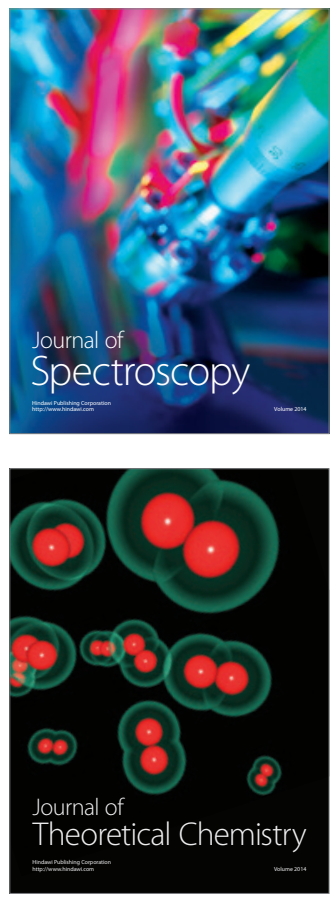
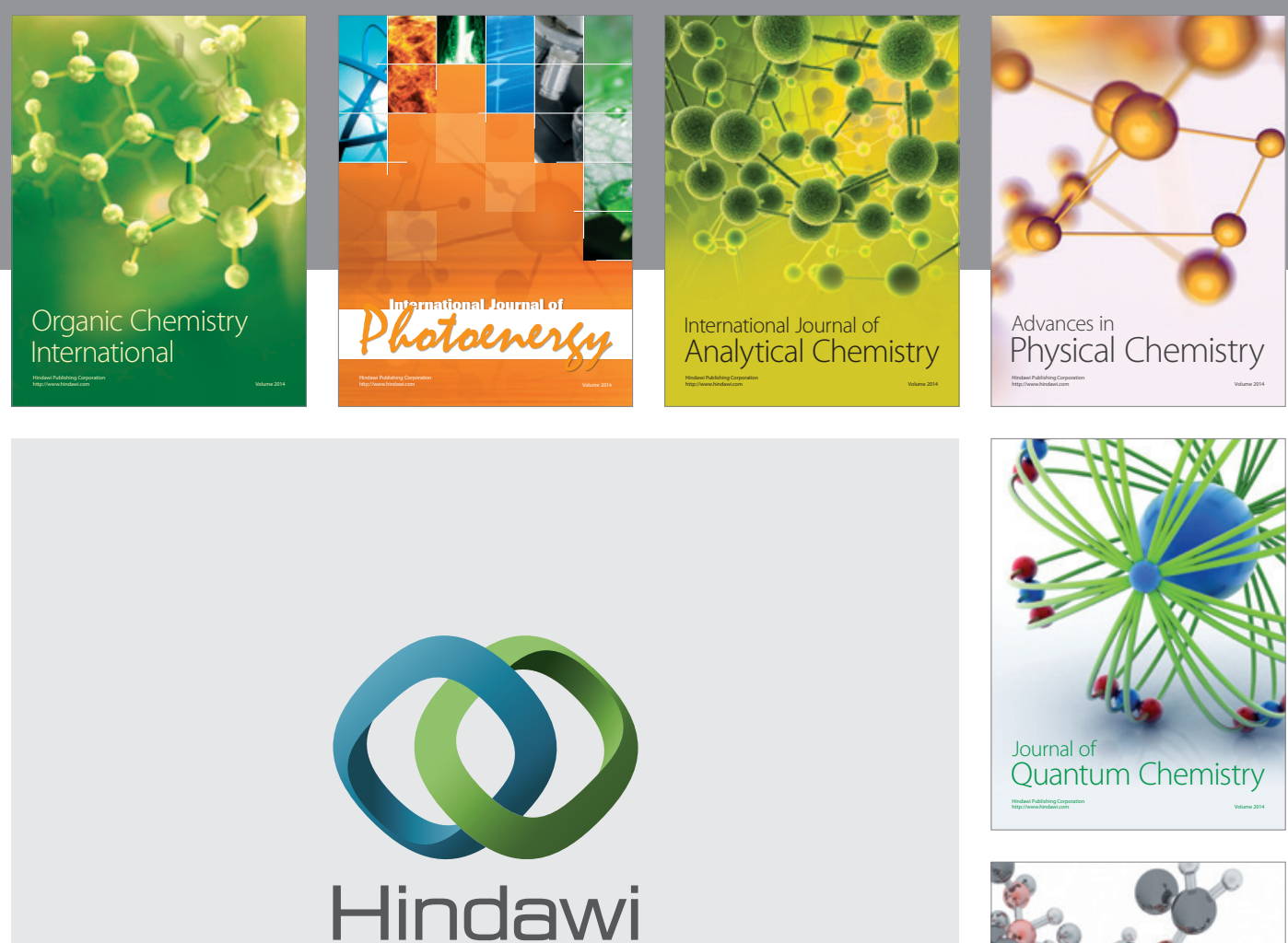

Submit your manuscripts at

http://www.hindawi.com

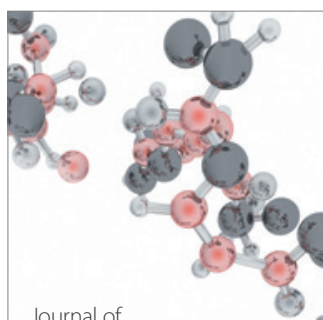

Analytical Methods

in Chemistry

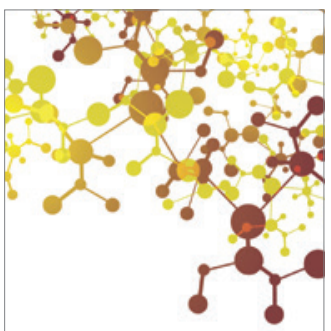

Journal of

Applied Chemistry

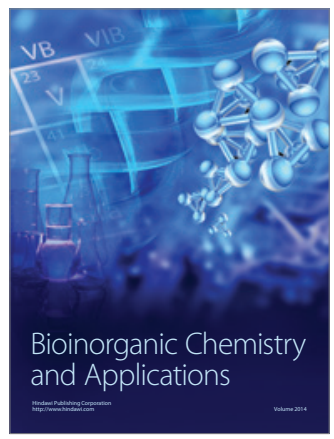

Inorganic Chemistry
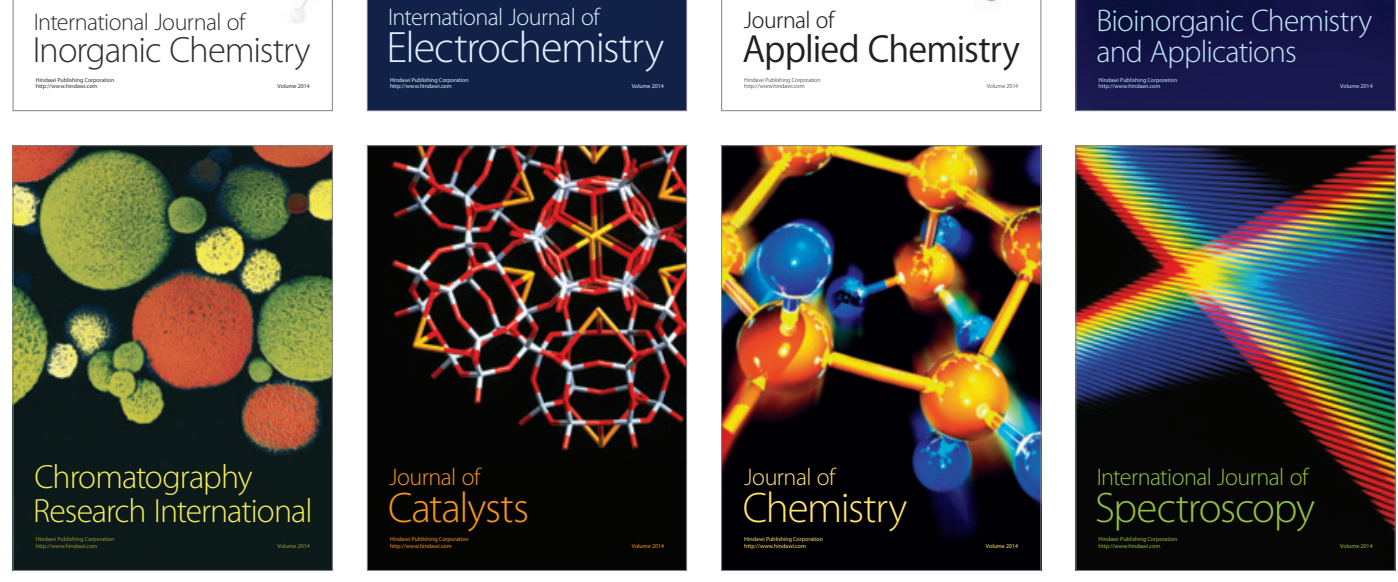